\title{
Catecholamine Response to Exercise in Children with Attention Deficit Hyperactivity Disorder
}

\author{
SHARON B. WIGAL, DAN NEMET, JAMES M. SWANSON, ROLAND REGINO, JOEY TRAMPUSH, \\ MICHAEL G. ZIEGLER, AND DAN M. COOPER
Child Development Center [S.B.W., J.M.S., R.R., J.T.], University of California, Irvine, Irvine, CA, 92612,
U.S.A.; Center for the Study of Health Effects of Exercise in Children [D.N., D.M.C.], University of
California, Irvine, College of Medicine, Orange, CA, 92686, U.S.A.; and Department of Medicine
[M.G.Z.], University of California, San Diego, San Diego, CA, 92103, U.S.A.

\begin{abstract}
The objective of this study was to examine differences in catecholamine (CA) response to exercise between children who had received a diagnosis of attention-deficit/hyperactivity disorder (ADHD) and age- and gender-matched controls. On the basis of the notion of a CA dysfunction in ADHD, we reasoned that the normal robust increase in circulating $\mathrm{CA}$ seen in response to exercise would be blunted in children with ADHD. To test this, we recruited 10 treatment-naïve children with newly diagnosed ADHD and 8 age-matched controls (all male) and measured CA response to an exercise test in which the work was scaled to each subject's physical capability. After exercise, epinephrine and norepinephrine increased in both control and ADHD subjects ( $p$ $=0.006$ and $p=0.002$, respectively), but the responses were substantially blunted in the ADHD group $(p=0.018)$ even though the work performed did not differ from controls. Circulating dopamine increased significantly in the control subjects ( $p$ $<0.016$ ), but no increase was noted in the subjects with ADHD.
\end{abstract}

\section{ABSTRACT}

Finally, a significant attenuation in the lactate response to exercise was found in ADHD (between groups, $p<0.005$ ). Our data suggest that $\mathrm{CA}$ excretion after exercise challenges in children with ADHD is deficient. This deficiency can be detected using a minimally invasive, nonpharmacologic challenge. (Pediatr Res 53: 756-761, 2003)

$\quad$ Abbreviations
ADHD, attention-deficit/hyperactivity disorder
CA, catecholamine
NE, norepinephrine
EPI, epinephrine
DA, dopamine
HPA, hypothalamic-pituitary-adrenal
BMI, body mass index
VO, oxygen consumption
CV, coefficients of variation

A leading pathophysiologic hypothesis of attention-deficit/ hyperactivity disorder (ADHD) is based on the notion of a catecholamine $[\mathrm{CA}$; norepinephrine $(\mathrm{NE})$, epinephrine (EPI), and dopamine (DA)] dysfunction $(1,2)$. This hypothesis suggests that the CA response to environmental stimuli is attenuated in ADHD and is derived primarily from observations that drugs such as methylphenidate and amphetamine-considered to be CA agonists - are effective in treating the symptoms of ADHD (1). Despite this compelling evidence, a definitive role of CA responsiveness in ADHD remains controversial (3).

Received July 30, 2002; accepted January 6, 2003.

S.B.W. and D.N. contributed equally to this work.

Correspondence: Dan M. Cooper, M.D., Professor of Pediatrics-University of California, Irvine, College of Medicine, Clinical Research Center, Bldg 25, ZOT 4094-03, 101 The City Drive, Orange, CA 92868, U.S.A.; e-mail: dcooper@uci.edu

This work was supported by grants MO1-RR00827, HD 23969, and MH02042 from the National Institutes of Health and by the Susan Samueli Center for Complementary and Alternative Medicine. D.N. is a postdoctoral research fellow of the Joseph W. Drown Foundation.

DOI: 10.1203/01.PDR.0000061750.71168.23
Testing CA responsiveness in children with ADHD has proved to be complex. Protocols that elicit psychological stress using cognitive challenges - the bulk of research that has been done in children with ADHD_can yield measurable CA responses, but the stimulus is difficult to quantify or standardize $(4,5)$. Pharmacologic interventions that stimulate stress through, for example, rapid alterations in glycemia, are nonphysiologic, require extensive monitoring, and may not be acceptable or feasible for studies in children (6).

In the present study, we examined the possibility that exercise testing might be useful in differentiating CA responses to stress between subjects who have a diagnosis of ADHD and age- and gender-matched controls. Physical activity is widely known to be a powerful stimulus of the hypothalamic-pituitaryadrenal (HPA) and noradrenergic systems (7). Physical and mental stress each elicits physiologic responses that are mediated through the autonomic nervous system and endocrine system (8). In contrast to other types of stress-inducing protocols, exercise is a naturally occurring and physiologic stimulus of stress hormones, and the magnitude of the input (i.e. the 
work rate and duration) can be measured precisely and scaled to the capability of the subject. To our knowledge, testing the $\mathrm{CA}$ response using exercise has never been reported in children with ADHD.

We reasoned that the normal robust increase in circulating $\mathrm{CA}$ in response to exercise would be blunted in children with ADHD. To test this, we recruited treatment naïve children with newly diagnosed ADHD and measured CA response to an exercise test in which the work was scaled to each subject's physical capability.

\section{METHODS}

The study was approved by the Institutional Review Board of the University of California, Irvine. Informed assent and consent were obtained from each subject and his or her parent or legally authorized representative, respectively, before the implementation of any study-related procedures. Standard, calibrated scales and stadiometers were used to determine height, weight, body mass index (BMI; wt $\left./ \mathrm{ht}^{2}\right)$, and BMI-for-age percentile (9).

Diagnosis of ADHD. Families were recruited by a screening study for evaluation of children with ADHD at the University of California, Irvine, Child Development Center. Children between the ages of 7 and 12 y were eligible. For inclusion in the ADHD group, a diagnosis of ADHD-combined hyperactive/impulsive subtypes was required. This was confirmed in a psychiatric interview of the parent about the child, by endorsement of at least six of the nine symptoms of inattention and six of the nine symptoms of hyperactivity/impulsivity on the Diagnostic Interview Schedule for Children. Children with a current history of depression, anxiety, epilepsy, or other medical conditions were excluded. All children who entered the study were naïve with respect to the use of stimulant medications to treat ADHD. Gender- and age-matched children who were healthy and had no history of ADHD were recruited as a control group.

Exercise protocols. We used an exercise that has been found to be effective in scaling the exercise input to the capabilities of healthy children as well as children with physiologic impairments (10). Each subject underwent two separate exercise testing sessions performed on different days within a week. First, we used a ramp-type progressive exercise test on an electronically braked cycle ergometer used extensively in children and adolescents (11). The second session consisted of a series of 10, 2-min bouts of constant-work rate cycle ergometry with 1-min resting intervals between each exercise bout. The work rate was individualized for each subject by finding the work rate corresponding to $50 \%$ of the difference between the anaerobic or lactate threshold of each subject (determined noninvasively from the ramp test) and the peak oxygen consumption $\left(\mathrm{VO}_{2}\right)(12,13)$. This approach was used, in contrast to the prolonged exercise testing, because although young children enjoy prolonged periods of physical activity, they find it hard to sustain constant exercise for more than several minutes at a time. In fact, typical bouts of exercise in children last only $25-30 \mathrm{~s} \mathrm{(14).} \mathrm{The} \mathrm{total} \mathrm{duration} \mathrm{of} \mathrm{the} \mathrm{second} \mathrm{exercise}$ protocol was $30 \mathrm{~min}$ (20 $\mathrm{min}$ of cycle ergometer exercise interspersed with 10 min of rest).

We calculated total external work performed by each subject [i.e. power $\times$ duration (kilojoules)] and normalized the total work performed to body mass. Finally, we measured the peak and end-exercise heart rate of each subject during the second exercise session.

Blood sampling. An indwelling venous catheter was inserted in the antecubital area. Blood samples were collected at pre-exercise (after $30 \mathrm{~min}$ of rest), during the last (tenth) 2-min exercise bout, 30 and 60 min after exercise.

$\boldsymbol{E P I}, \boldsymbol{N E}$, and $\boldsymbol{D A}$. EPI, NE, and DA were measured by a radioenzymatic technique based on the conversion of the $\mathrm{CA}$ to radiolabeled metanephrine and normetanephrine. This CA assay uses an extraction technique that eliminates substances that may inhibit the radioenzymatic assay. It also concentrates the CA to provide a more sensitive assay. One milliliter of plasma samples was extracted and then concentrated into a $0.1-\mathrm{mL}$ volume before conversion into their radiolabeled metabolites. The assay has an extraction efficiency of $78 \%$. The sensitivity of the assay is 10 and $6 \mathrm{pg} / \mathrm{mL}$ for NE and EPI. The intra-assay coefficients of variation (CV) are 4 and $13 \%$ for samples containing low levels of CA; variation is less for samples with high levels of CA. The inter-assay CV are $10 \%$ and $16 \%$, respectively, for NE and EPI, so the assay is consistent over time. This technique is approximately 10 times more sensitive than the more commonly used assays and thus can reveal changes in venous CA levels that often go undetected (15).

Lactate. Lactate was measured with the use of YSI lactate analyzer (YSI 1500, Yellow Springs, OH, U.S.A.). The intraassay $\mathrm{CV}$ was $2.8 \%$, the interassay $\mathrm{CV}$ was $3.5 \%$, and the sensitivity was $0.2 \mathrm{mg} / \mathrm{dL}$.

Statistical analysis. Two-sample $t$ tests were used to determine baseline differences in anthropometric variables, fitness variables, and circulating $\mathrm{CA}$ between control subjects and subjects with ADHD before the exercise protocol. Repeated measures ANOVA was used to test differences in response to the exercise bout between ADHD and control tests groups. For detecting possible differences in the pattern of response to exercise over time, the primary test of interest was the interaction of the between-subjects factor (group: ADHD versus control) and the within-subject factor (time: before, peak, 30 min after, and $60 \mathrm{~min}$ after). A post hoc single degree of freedom contrast to compare the baseline to peak change by group was tested to characterize whether the magnitude of response differed between the groups. Data are presented as mean \pm SEM.

\section{RESULTS}

\section{Baseline Demographic Data}

Ten newly diagnosed untreated male subjects (eight Caucasian, two Hispanic) with ADHD and eight healthy age-matched male controls (seven Caucasian, one Hispanic) volunteered for the study and met the screening criteria. Subject characteristics are presented in Table 1. No significant differences in age, height, weight, or BMI were found between control and ADHD groups. 


\section{Effect of Brief Exercise}

Peak $\mathrm{VO}_{2}$, work rate, and heart rate. No significant differences were found in peak $\mathrm{VO}_{2}$, peak $\mathrm{VO}_{2}$ corrected for body weight, peak work rate, and lactate threshold between control subjects and subjects with ADHD (Table 1). The work rate performed per kilogram of body weight $(\mathrm{kJ} / \mathrm{kg})$ was almost identical in the ADHD and control groups. The control group reached a higher heart rate by end-exercise compared with the subjects with ADHD, but this difference was not significant $(189.4 \pm 3.1$ versus $178.1 \pm 5.1$ respectively; Fig. 1$)$.

Plasma lactate and $\boldsymbol{C A}$. Plasma lactate increased significantly during exercise in both control and ADHD groups $(p<$ $0.001)$. There was a significant between-group difference in the lactate response to exercise, with a more prominent change in the control group ( $p<0.005$; Fig. 2).

Baseline levels of NE and EPI were within the normal range for both children with ADHD and controls, suggesting that the blood drawing technique/timing was not stressful. Baseline plasma NE were significantly lower in the ADHD children ( $p$ $=0.004)$. In response to exercise, mean NE levels rose in both groups; however, the rise in plasma NE was significantly greater in the control children compared with children with ADHD, reaching levels that were more than 2-fold higher in the control group ( $p<0.0005$; Fig. 3).

No difference was found for baseline plasma EPI. EPI levels increased in both $\operatorname{ADHD}(p=0.002)$ and controls $(p=0.006)$ after exercise. A statistically significant higher level of EPI at peak exercise was found in the control group $(p=0.018)$. Baseline plasma levels of DA tended to be higher in the ADHD group, but this difference was not significant. After exercise, DA levels in the ADHD group did not change, whereas a significant increase was noted in the control group $(p=0.016)$.

\section{DISCUSSION}

This study demonstrates for the first time abnormal responses of circulating EPI, NE, and DA accompanying cycle ergometer exercise in treatment-naïve children with newly diagnosed ADHD. EPI and NE did increase in both control subjects and subjects with ADHD, but the responses were substantially blunted in the ADHD group even though the work performed did not differ from controls. Circulating DA increased significantly in the control subjects, but no increase was noted in the subjects with ADHD. Finally, a significant
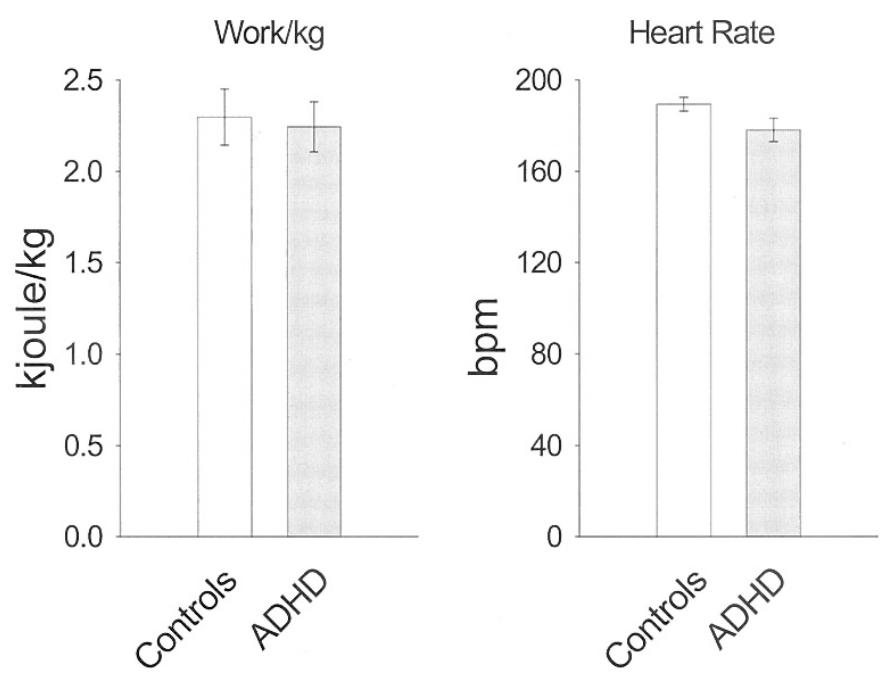

Figure 1. Comparison among total work per body weight and peak heart rate in control subjects and subjects with ADHD. Both ADHD and control groups performed the same total work per body weight. Peak heart rate was higher in the control group but not significantly so.

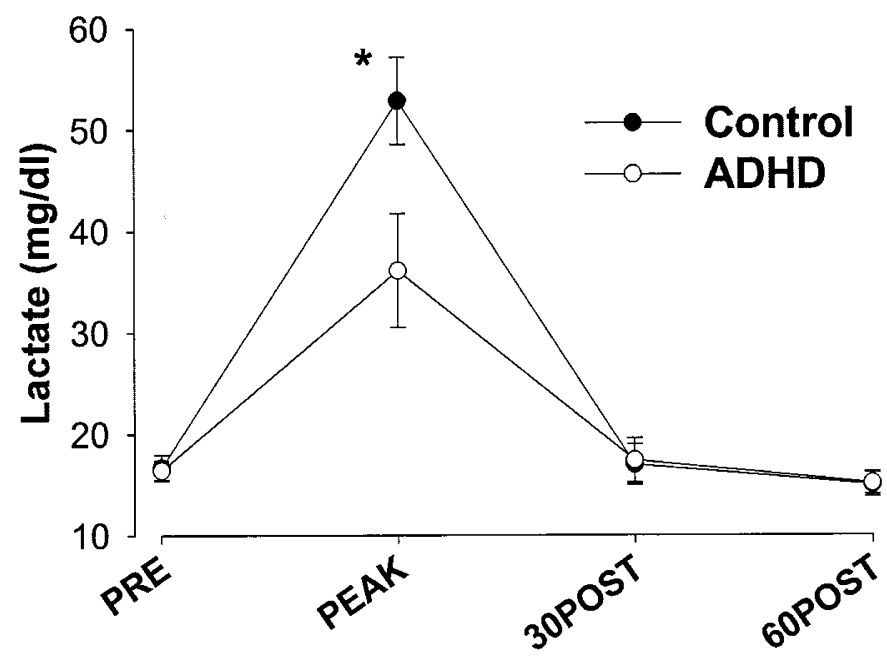

Figure 2. The effect of exercise on lactate levels. Both control subjects and subjects with ADHD had an increase in lactate level after exercise. Control subjects had a statistically significant higher lactate response to exercise compared with subjects with ADHD $(* p<0.005)$.

lower lactate response to exercise was found in ADHD, an observation consistent with a blunted CA response to exercise.

Table 1. Anthropometric, peak $\dot{V} O_{2}$, and peak work rate in subjects with ADHD and control subjects

\begin{tabular}{|c|c|c|}
\hline & $\operatorname{ADHD}(n=10)$ & Control $(n=8)$ \\
\hline Age (years) & $8.4 \pm 0.4($ range $7-10)$ & $8.6 \pm 0.5($ range $7-11)$ \\
\hline Height $(\mathrm{cm})$ & $135 \pm 3($ range $117-145)$ & $138 \pm 3($ range $127-154)$ \\
\hline Weight (kg) & $33.8 \pm 2.8($ range $22.3-52.7)$ & $34.4 \pm 2.8$ (range $25.0-48.6)$ \\
\hline Peak $\mathrm{VO}_{2}(\mathrm{~L} / \mathrm{min})$-ramp test & $1.01 \pm 0.07$ (range $0.47-1.34)$ & $1.11 \pm 0.09($ range $0.83-1.48)$ \\
\hline Peak $\mathrm{VO}_{2}\left(\mathrm{~mL} \cdot \min ^{-1} \cdot \mathrm{kg}^{-1}\right)$-ramp test & $30.2 \pm 1.7($ range $21.2-37.2)$ & $32.8 \pm 2.0($ range $24.4-41.6)$ \\
\hline Lactate threshold $(\mathrm{L} / \mathrm{min})$ & $0.78 \pm 0.06$ (range $0.48-1.03)$ & $0.79 \pm 0.05($ range $0.62-1.09)$ \\
\hline Heart rate during constant work rate protocol (beats/min) & $152.1 \pm 4.6($ range $124-172)$ & $159.9 \pm 7.1($ range $136-189)$ \\
\hline
\end{tabular}

Data are presented as mean \pm SEM. 
$\multimap-$ ADHD
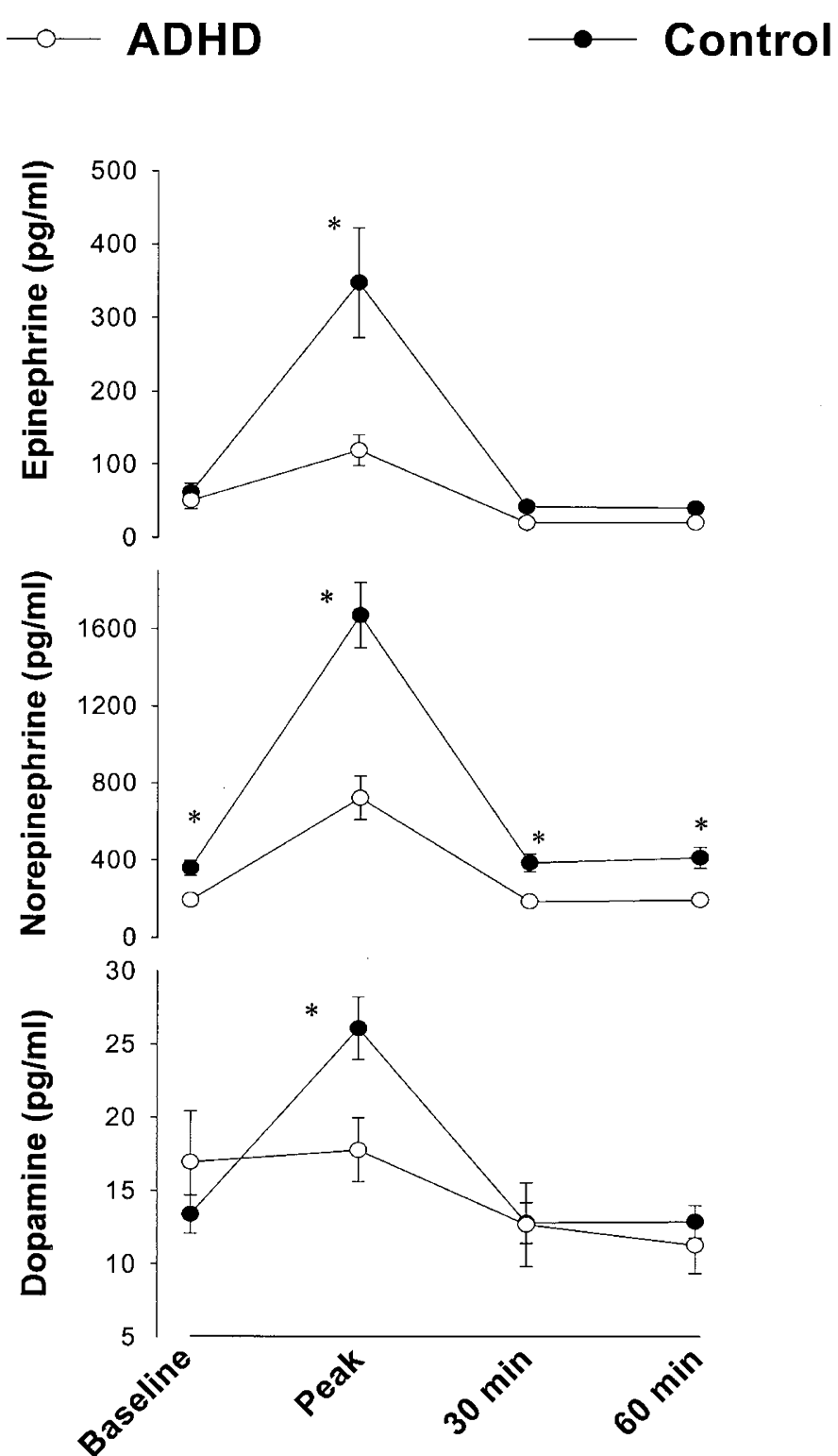

Figure 3. The effect of exercise on CA levels. Significant differences in EPI, $\mathrm{NE}$, and DA responses to exercise were found between the control subjects and subjects with ADHD (*p<0.05).

We selected a relatively high-intensity exercise protocol for this study because the CA response for work performed above the lactate threshold is known to be substantial (16). CA are increased with heavy exercise, in part because of CNS mechanisms. Activation of the HPA axis and sympathetic-adrenalmedullary activation leads to EPI release from the adrenal medulla and NE and, to a lesser degree, DA release, from nerve endings into the circulation $(17,18)$. Thus, exercise shares with other stresses (e.g. psychosocial) some common pathways that lead to increased CA output. In addition, the CA response to heavy exercise is further stimulated by systemic changes in acid-base balance and reduced oxygen availability to the working tissues (19).

Remarkably, the increase in circulating DA in response to exercise found in healthy children was absent in the subjects with ADHD (Fig. 3). Previous studies have demonstrated an increase in circulating DA in response to cycle ergometer (20) and resistance exercise (21) in adults, but this is the first documentation of the increase in circulating DA in healthy children. Whether the lack of a DA response in the periphery in ADHD is related, as some investigators propose, to a systemic "dopamine deficit" $(22,23)$ or, alternatively, simply to less stimulation of the adrenals in response to exercise has yet to be determined.

The present data provide indirect support for the connection between exercise and stimulation of HPA and noradrenergic systems in children with ADHD. This is in agreement with previous studies, for example, Hanna and et al. (24), Pliszka et al. (25), and Anderson et al. (4) who found substantially lower rates of EPI excretion in urine during cognitive testing in subjects with ADHD. These observations are consistent with earlier studies correlating academic performance and EPI excretion (26).

Most consistent with our observation of a blunted CA response to a physiologic stress in ADHD is the study of Girardi et al. (6). These investigators gave subjects an oral glucose load that led to an initial hyperglycemia followed by a rapid lowering of blood glucose and an accompanying CA burst. As in our study, Girardi et al. noted a blunted CA response in children who had a diagnosis of ADHD.

In studies that use exercise as an input to stimulate hormonal responses, a major potential confounding factor is whether the exercise input is comparable in the control and target groups. For example, if a protocol used a single work rate to compare hormonal response to exercise in two groups of subjects with high and low relative fitness, then the results might be confounded by the fact that the magnitude of the exercise input relative to the capability of each subject was different in the two groups (i.e. relatively higher in the unfit sample population). We achieved the goal of appropriately normalizing the work rates in the two populations by measuring fitness in each subject and adjusting the work rate input to the individual's capabilities. As seen in Figure 1, we found no difference in the magnitude of the work rate input (either in absolute watts or when normalized to body mass) between the two groups. Thus, it is unlikely that the observed difference in the CA response was due to a lower relative work rate input in the ADHD group; rather, the data suggest abnormal CA regulation in the subjects with ADHD.

It is noteworthy that the peak $\mathrm{VO}_{2}$ values observed in our study tended to be in the lower range of normal values that are typically reported for cycle ergometer exercise in children. Although a strict matched-control design was not used, we sought the control group for this study from a general population and did not target children actively engaged in physical activity, as is often the case, for "normal" values in studies of exercise in children. It may well be and would not be surprising that in a large population comparison between healthy control children and children with ADHD that the latter would prove to have significantly lower levels of fitness.

A number of studies have been performed in normal subjects in which centrally acting pharmacologic agents were used to alter the CA response to exercise. Collomp et al. (27) and Stratton et al. (28) showed that benzodiaz- 
epines, which stimulate $\gamma$-aminobutyric acid receptors in the brain and blunt the CA response to a variety of stressors, markedly attenuated the EPI, NE, and DA response to exercise in the circulation. Interestingly, these investigators found that benzodiazepines attenuated the lactate response to exercise, similar to the observation we made in the children with ADHD (Fig. 2), and the blunted lactate response is likely explained by the reduced effect of peripheral CA on glucose metabolism.

Although the lactate levels in response to exercise were lower, the lactate threshold (the inflection point above which lactate concentrations in the circulation markedly increase) was not affected in the Stratton study. Similarly, we found no difference in the lactate threshold, determined noninvasively, between the subjects with ADHD and control subjects. These data showing that CNS suppression can lead to blunted peripheral CA responses supports the idea that there may exist a CNS dysregulation of CA in children with ADHD.

In addition to the exercise response, we found decreased baseline NE in the patients with ADHD. Surprising, little is known about circulating levels of CA in ADHD as the majority of the studies have focused on urine CA levels $(2,4)$. The lower NE that we found is consistent with some (29-31) but not all (32) previous studies. In the past, some investigators have questioned the relevance of peripheral (i.e. circulating) levels of CA in that they may not reflect CNS activity. However, it has become clear that activity of the peripheral nervous system does correlate with activity in the brain (33). Indeed, administering $\mathrm{CA}$ into the peripheral circulation (n.b., CA reportedly do not cross the blood-brain barrier) induce cognitive changes strongly suggestive of CNS effects (34).

Both physical exercise (35) and traditional pharmacologic treatment for ADHD with low doses of methylphenidate or amphetamine (36) increase executive function of the brain. Shepard et al. (37) demonstrated that long-term increases in physical activity were associated with improved academic performance in public school students. Despite the compelling physiologic role that exercise could play in the management of ADHD, to our knowledge, there have been no controlled studies designed to examine potential therapeutic benefits of exercise in ADHD. Our data pose some intriguing questions. First, does the blunted CA response to exercise also reflect a reduced exercise effect on executive function? Does repeated exercise (i.e. training) lead to an enhanced CA response to exercise or to other stresses in ADHD? Finally, does the CA response to exercise become normalized in the presence of traditional pharmacologic treatments with stimulant medications?

\section{CONCLUSION}

In summary, we found that the CA response to exercise was markedly reduced in children with ADHD. The agreement across studies examining the adrenomedullary and sympathetic responses in ADHD using various provocations, whether pharmacologic, cognitive, or physiologic, is remarkable. Our data suggest that $\mathrm{CA}$ excretion after a minimally invasive, nonpharmacologic exercise challenge in children with ADHD is defi- cient compared with healthy control children. These preliminary data are consistent with previous studies indicating that children with ADHD have lower CA responses to pharmacologic, physiologic, and cognitive challenges.

\section{REFERENCES}

1. Zametkin AJ, Rapoport JL 1987 Neurobiology of attention deficit disorder with hyperactivity: where have we come in 50 years? J Am Acad Child Adolesc Psychiatry 26:676-686

2. Pliszka SR, McCracken JT, Maas JW 1996 Catecholamines in attention-deficit hyperactivity disorder: current perspectives. J Am Acad Child Adolesc Psychiatry $35: 264-272$

3. Swanson JM, Sergeant JA, Taylor E, Sonuga-Barke EJ, Jensen PS, Cantwell DP 1998 Attention-deficit hyperactivity disorder and hyperkinetic disorder. Lancet 351:429433

4. Anderson GM, Dover MA, Yang BP, Holahan JM, Shaywitz SE, Marchione KE, Hall LM, Fletcher JM, Shaywitz BA 2000 Adrenomedullary function during cognitive testing in attention-deficit/hyperactivity disorder. J Am Acad Child Adolesc Psychiatry $39: 635-643$

5. King JA, Barkley RA, Barrett S 1998 Attention-deficit hyperactivity disorder and the stress response. Biol Psychiatry 44:72-74

6. Girardi NL, Shaywitz SE, Shaywitz BA, Marchione K, Fleischman SJ, Jones TW, Tamborlane WV 1995 Blunted catecholamine responses after glucose ingestion in children with attention deficit disorder. Pediatr Res 38:539-542

7. Wittert G 2000 The effect of exercise on the hypothalamo-pituitary-adrenal axis. In: Warren MP, Constantini NW (eds) Sports Endocrinology. Humana Press, Totowa, pp $43-55$

8. Wasmund WL, Westerholm EC, Watenpaugh DE, Wasmund SL, Smith ML 2002 Interactive effects of mental and physical stress on cardiovascular control. J Appl Physiol 92:1828-1834

9. Kuczmarski RJ, Ogden CL, Grummer-Strawn LM, Flegal KM, Guo SS, Wei R, Mei Z, Curtin LR, Roche AF, Johnson CL 2000 CDC growth charts: United States. Adv Data $314: 1-27$

10. Tirakitsoontorn P, Nussbaum E, Moser C, Hill M, Cooper DM 2001 Fitness, acute exercise, and anabolic and catabolic mediators in cystic fibrosis. Am J Respir Crit Care Med 164:1432-1437

11. Cooper DM, Weiler-Ravell D, Whipp BJ, Wasserman K 1984 Aerobic parameters of exercise as a function of body size during growth in children. J Appl Physiol $56: 628-634$

12. Armon Y, Cooper DM, Springer C, Barstow TJ, Rahimizadeh H, Landaw E, Epstein S 1990 Oral 13C-bicarbonate measurement of $\mathrm{CO}_{2}$ stores and dynamics in children and adults. J Appl Physiol 69:1754-1760

13. Barstow TJ, Cooper DM, Epstein S, Wasserman K 1989 Changes in breath ${ }^{13} \mathrm{CO}_{2} /$ ${ }^{12} \mathrm{CO}_{2}$ consequent to exercise and hypoxia. J Appl Physiol 66:936-942

14. Bailey RC, Olson J, Pepper SL, Barstow TJ, Porszsasz J, Cooper DM 1995 The level and tempo of children's physical activities: an observational study. Med Sci Sports Exerc 27:1033-1041

15. Kennedy B, Ziegler MG 1990 A more sensitive and specific radioenzymatic assay for catecholamines. Life Sci 47:2143-2153

16. Urhausen A, Weiler B, Coen B, Kindermann W 1994 Plasma catecholamines during endurance exercise of different intensities as related to the individual anaerobic threshold. Eur J Appl Physiol Occup Physiol 69:16-20

17. Pedersen BK, Hoffman-Goetz L 2000 Exercise and the immune system: regulation, integration, and adaptation. Physiol Rev 80:1055-1081

18. Van Loon GR 1983 Plasma dopamine: regulation and significance. Fed Proc 42:3012-3018

19. Schneider DA, McGuiggin ME, Kamimori GH 1992 A comparison of the blood lactate and plasma catecholamine thresholds in untrained male subjects. Int J Sports Med 13:562-566

20. Nagao F, Suzui M, Takeda K, Yagita H, Okumura K 2000 Mobilization of NK cells by exercise: downmodulation of adhesion molecules on NK cells by catecholamines. Am J Physiol Regul Integr Comp Physiol 279:R1251-R1256

21. Kraemer WJ, Fleck SJ, Maresh CM, Ratamess NA, Gordon SE, Goetz KL, Harman EA, Frykman PN, Volek JS, Mazzetti SA, Fry AC, Marchitelli LJ, Patton JF 1999 Acute hormonal responses to a single bout of heavy resistance exercise in trained power lifters and untrained men. Can J Appl Physiol 24:524-537

22. Levy F 1991 The dopamine theory of attention deficit hyperactivity disorder (ADHD). Aust N Z J Psychiatry 25:277-283

23. Volkow ND, Wang GJ, Fowler JS, Logan J, Franceschi D, Maynard L, Ding YS, Gatley SJ, Gifford A, Zhu W, Swanson JM 2002 Relationship between blockade of dopamine transporters by oral methylphenidate and the increases in extracellular dopamine: therapeutic implications. Synapse 43:181-187

24. Hanna GL, Ornitz EM, Hariharan M 1996 Urinary catecholamine excretion and behavioral differences in ADHD and normal boys. J Child Adolesc Psychopharmacol 6:63-73

25. Pliszka SR, Maas JW, Javors MA, Rogeness GA, Baker J 1994 Urinary catecholamines in attention-deficit hyperactivity disorder with and without comorbid anxiety. J Am Acad Child Adolesc Psychiatry 33:1165-1173

26. Frankenhaeuser M 1971 Behavior and circulating catecholamines. Brain Res 31:241262

27. Collomp K, Fortier M, Cooper S, Long A, Ahmaidi S, Prefaut C, Wright F, Picot M, Cote MG 1994 Performance and metabolic effects of benzodiazepine during submaximal exercise. J Appl Physiol 77:828-833 
28. Stratton JR, Halter JB 1985 Effect of a benzodiazepine (alprazolam) on plasma epinephrine and norepinephrine levels during exercise stress. Am J Cardiol 56:136 139

29. Shekim WO, Sinclair E, Glaser R, Horwitz E, Javaid J, Bylund DB 1987 Norepinephrine and dopamine metabolites and educational variables in boys with attention deficit disorder and hyperactivity. J Child Neurol 2:50-56

30. Shekim WO, Javaid J, Dekirmenjian H, Chapel JL, Davis JM 1982 Effects of d-amphetamine on urinary metabolites of dopamine and norepinephrine in hyperactive boys. Am J Psychiatry 139:485-488

31. Shekim WO, Dekirmenjian H, Chapel JL 1978 Urinary MHPG excretion in the hyperactive child syndrome and the effects of dextroamphetamine. Psychopharmacol Bull 14:42-44

32. Brown GL, Ebert MH, Minichiello MD 1985 Biochemical and pharmacological aspects of attention deficit disorder. In: LM Bloomingdale (ed) Attention Deficit Disorder: Identification, Course, and Rationale. Spectrum Press Medical and Scientific Books, New York, pp 93-130
33. Maas JW, Leckman JF 1983 Relationship between central nervous system noradrenergic function and plasma and urinary MHPG and other norepinephrine metabolites. In: Maas JW (ed) MHPG: Basic Mechanisms and Psychopathology. Academic Press, London, pp 33-44

34. van Zijderveld GA, van Doornen LJ, van Faassen I, Orlebeke JF, van Dyck R, Tilders FJ 1993 Adrenaline and the relationship between neurosomatism, aerobic fitness and mental task performance. Biol Psychol 36:157-181

35. Tantillo M, Kesick CM, Hynd GW, Dishman RK 2002 The effects of exercise on children with attention-deficit hyperactivity disorder. Med Sci Sports Exerc 34:203212

36. Solanto MV 1998 Neuropsychopharmacological mechanisms of stimulant drug action in attention-deficit hyperactivity disorder: a review and integration. Behav Brain Res 94:127-152

37. Shephard RJ 1996 Habitual physical activity and academic performance. Nutr Rev $54: \mathrm{S} 32-\mathrm{S} 36$ 